\title{
Research on Long-Chain Non-Coding RNA and Breast Cancer: A Review
}

\author{
Arabela N. Gerardi, Igor E. Sullivan \\ Louisiana State University, Louisiana 70803, USA.
}

\begin{abstract}
How to cite this paper: Arabela N. Gerardi, Igor E. Sullivan. (2020) Research on Long-Chain Non-Coding RNA and Breast Cancer: A Review. International Journal of Clinical and Experimental Medicine Research, 4(3), 47-52.

DOI: 10.26855/ijcemr.2020.07.003
\end{abstract}

Received: April 25, 2020

Accepted: May 30, 2020

Published: June 24, 2020

\begin{abstract}
lncRNAs play an important role in the occurrence, metastasis, treatment and prognosis of various cancers including breast cancer. Therefore, lncRNAs have become a hot topic in cancer research. At present, several common lncRNAs, such as HOTAIR, H19, GAS5, MALAT1, LSINCT5, and SRA, have been extensively studied, and their mechanisms in breast tumors have gradually become clear as the research progresses. However, the research of IncRNAs such as LincROR, UCA1, MEG3, and NKILA is still at an early stage, and more research is needed to clarify its mechanism in cancer. With the study of lncRNAs, more lncRNAs will be discovered. Although our current understanding on the molecular mechanism of lncRNA in various biological processes is still limited, we hope it can be used for tumor diagnosis, treatment and prognosis for patients with breast cancer in future clinic work.
\end{abstract}

\section{Keywords}

long-chain noncoding RNA, breast cancer, diagnosis, treatment, expression

\section{Introduction}

Breast cancer is the most common cancer in Chinese women, and it is also the main cause of cancer death [1]. In 2015, Chinese women are expected to have about 4.292 million new breast cancer patients, accounting for 15\% of all new cancer women [2]. At present, there are many studies on the etiology of breast cancer, but the specific pathogenesis is not clear. However, epidemiological studies have shown that the development of breast cancer is usually caused by the interaction of genetic, environmental, lifestyle and reproductive risk factors [3-5].

At present, it is believed that at least $98 \%$ of mammalian and other complex organisms' genomes are transcribed into noncoding RNA [6]. Recent studies have revealed the existence of a wide range of noncoding RNA (ncRNAs) and provided convincing evidence for the regulatory role of ncRNAs in certain pathological and disease processes [7-9]. In addition, the number of non-coding RNA in sperm genome is more consistent with the complexity of organisms compared with the number of coding genes, which indicates that the regulatory mechanism based on RNA is the key to the complexity of biological evolution [10]. In addition to the well-known transport RNA (tRNA), ribosomal RNAs (rRNA) and small nuclear RNAs (snoRNAs), the family of noncoding RNAs also includes the recently discovered long-chain noncoding RNAs (lncrnas) and miRNAs. A large number of studies have emphasized the regulatory role of miRNAs in cancer development and the relationship between their gene polymorphisms and various cancer risks [11,12]. In addition to miRNAs, more and more studies have found that lncRNAs play an important role in various biological processes from embryonic development to human diseases, including cell cycle control, apoptosis, invasion and migration [13]. The differential expression of several lncRNAs in different tumors also indicates the close relationship between lncRNA and carcinogenesis [14-16]. The role of Incrnas in tumorigenesis and development has attracted more and more attention, which has 
become a hot topic in the field of molecular biology.

\section{LncRNA}

LncRNAs are endogenous RNA molecules with a length of more than 200 bases but without protein coding function [17]. According to the location of the gene and the direction of transcription, it can be further divided into synonymy (the same as the template chain of protein gene transcription), antisense (complementary to the template chain of protein gene transcription), bidirectional (it comes from two reverse complementary chains of protein gene, usually the distance between transcription starting sites is less than 1,000 BP), intron (its template chain of transcription located in the intron sequence of the coding protein gene) and the intergenic lncrnas (whose transcription template chain is located in the gene interval region of the coding protein) [18]. Most lncRNAs have similar transcription mechanism (usually generated by RNA polymerase II transcription), epigenetic regulation (such as histone modification profile) and splicing signal with mRNA [19, 20]. Due to the lack of open reading frame and poor sequence conservation, lncRNA has been considered as a non-functional gene "transcription noise" in the past. Recent studies have found that lncrnas may contain short open reading frames (sORF, less than 100 codons) that can produce small peptides, which are often ignored in the past when predicting open reading frames because the length of the codon is less than 100 [21, 22]. LncRNAs can play a role in both nucleus and cytoplasm. LncRNAs of nucleus participate in chromatin remodeling and modification, transcription regulation and RNA processing, while lncRNAs in cytoplasm usually interact with mature mRNA or protein [23].

With deeper development of lncRNAs research, more and more functions of lncRNAs have been revealed. More and more evidences show that abnormal expression of lncRNAs may play an important role in cancer biology [24]. Based on a lot of studies related to lncrnas, the extensive effects of lncRNAs on various physiological processes of cells are summarized as follows: 1) interference with gene transcription; 2) regulation of chromatin rearrangement and histone modification; 3) interference with mRNA cutting; 4) change of protein function; 5) subcellular localization of regulatory proteins; 6) as competitive endogenous RNA [17].

\section{LncRNA and breast cancer}

More and more lncRNAs have been identified not only to participate in physiological processes but also to play a role in tumorigenesis, proliferation, apoptosis, and invasion. Some lncRNAs are even specifically associated with a tumor type. For example, SCHLAP1 overexpression is closely related to prostate cancer metastasis and prognosis, and HULC can be used as a biomarker for liver cancer diagnosis. Although personalized treatment based on its four molecular types (Luminal A, Luminal B, Her2 positive, and triple negative breast cancer) has made great progress in breast cancer, we still cannot reduce the high incidence of breast cancer and total mortality. Recently, studies have reported that some lncRNAs are closely related to breast cancer. In this review, we summarize the biological functions of long-chain non-coding RNAs related to breast cancer and their mechanisms of action. We hope to provide theoretical basis for exploring new treatments for breast cancer at the LncRNA molecular level.

\subsection{HOTAIR}

HOTAIR is a 2.2kb carcinogenic RNA located in mammalian homeobox C (HOXC) with chromosome position of 12q13.13. HOTAIR was first discovered in 2007 to inhibit transcription of the HOXD locus on chromosome 2 [25]. In 2010, Gupta et al. found that primary breast cancer tissues contained higher levels of HOTAIR than adjacent tissues [26]. HOTAIR was soon discovered to promote cancer cell metastasis and could be used as a biomarker to predict the prognosis of breast cancer [27]. Studies on the mechanism of HOTAIR have shown that HOTAIR can be used as a scaffold to bind functional complexes in several different fields. The 5 terminal of HOTAIR interacts with polycomb inhibitory complex 2 (PRC2) (including subunits such as SUZ12 and EZH2) to promote methylation of H3K27. Through this interaction, HOTAIR can silence its target genes to achieve gene regulation. In addition, the $3^{\prime}$ end of HOTAIR interacts with the LSD1/CoREST/REST complex to demethylate H3K4 to achieve the purpose of activating genes [28]. HOTAIR can affect the expression of multiple downstream genes by modifying specific histones [25]. For example, Gupta et al. [26] found that high expression of HOTAIR caused a decrease in PRC2 levels in 854 genes and thus altered gene expression. Gene ontology analysis also revealed that most of these genes are involved in cell signal transduction pathways.

The association between HOTAIR SNPs and genetic susceptibility to breast cancer has also been reported. A case-control study in Turkey showed that the CC genotype of HOTAIR rs920778 increased the risk of breast 
cancer (OR: 2.12; 95\% CI: 1.00-4.54) [29]. Studies by Yan et al. [30] showed that HOTAIR rs920778T allele could increase the risk of breast cancer. Such inconsistent results may be cased by the differences in race, sample size, and research methods in the two studies. Therefore, further large-scale studies need to determine the association between HOTAIR SNPs and genetic susceptibility to breast cancer.

\section{$3.2 \mathrm{H19}$}

H19 was one of the first genes to be found to play an important role in genomic imprinting. H19 is 2.3 is located within $200 \mathrm{~kb}$ downstream of the insulin-like growth factor 2 (IGF-2) gene with the length of 2.3kb, and has a chromosome position of $11 \mathrm{p} 15.5$ [31]. H19 is involved in the development of many cancers, including esophageal cancer [32], liver cancer [33], and breast cancer [34]. Researchers have found that lncRNA H19 expression is upregulated in breast cancer tissue or breast cancer cells. Oncogene protein (c-myc) is widely expressed in breast cancer tissues and can directly induce H19 transcription through allele-specific binding. The E2F1 factor regulates the progress of the cell cycle by binding to the promoter of the H19 gene, especially the transition from the G1 to S phase of the cell cycle [35]. A study on hepatocellular carcinoma showed that the most dangerous carcinogen (aflatoxin B1) could induce the expression of E2F1, and then up-regulate the expression of H19 directly or indirectly to promote cell growth and invasion [36]. In addition, lncRNA H19 has been confirmed as a precursor of miR-675, and the direct target gene of miR-675 is the tumor suppressor gene of retinoblastoma (Rb). H19 can indirectly affect the regulation of Rb in primary colorectal cancer [37]. However, researchers have not confirmedthe role of miR-675 in breast cancer. As research progresses, more and more H19's role in breast cancer have been revealed. In short, the overexpression of H19 is involved in the induction of breast cancer. siRNA treatment cased be used to inhibit the expression of H19 and prevent breast cancer cell proliferation. As an oncogene, H19 overexpression promotes the proliferation of breast cancer cell line MDA-MB-231. H19 single nucleotide polymorphisms are closely related to genetic susceptibility to various diseases. However, the association between H19 SNPs and breast cancer has been rarely reported.

\subsection{GAS5}

GAS5 was originally isolated from NIH3T3 cells, located on chromosome 1q25.1, and is about 0.6 to $1.8 \mathrm{~kb}$ in length. Its intron sequence encodes a variety of small nucleolar RNAs, while the exon sequence produces LncRNAGAS5. GAS5 is a tumor suppressor gene. Studies have found that GAS5 is down regulated in a variety of cancers, including breast cancer, prostate cancer, colorectal cancer, and lung cancer [38]. GAS5 can regulate apoptosis and differentiation of mammalian cells. Studies have shown that the GAS5 expression in breast cancer patient is reduced by at least 65\% compared to normal breast epithelial tissues. The differential expression of GAS5 in breast cancer stageI and II is obvious and different, suggesting that decreased GAS5 expression is an early event of cancer development and can be used to predict the stage of breast cancer [39].

Decreased GAS5 expression reduces the cell death of drug-induced breast cancer. The regulatory effect of GAS5 on apoptosis has been recognized as a possible explanation for chemotherapy resistance of cancer cells. In order to solve the problem of chemotherapy resistance of cancer cells, Pickard et al. Suggested to use drugs to bypass the GAS5 pathway or use PI3K/mTOR to increase GAS5 levels [40]. Another mechanism of GAS5 as a tumor suppressor gene is mutual inhibition with miR-21, miR-21 is highly expressed in many tumor tissues and can regulate tumorigenesis and metastasis [41]. First, GAS5 can bind to miR-21 exon 4, and silenced miR-21 can inhibit the transcription of several downstream genes, including PTEN, TDM1, and PCDC4. Second, miR-21 may down-regulate GAS5 expression by RISC in breast tumor specimens [42].

\subsection{MALAT1}

MALAT1 was originally found to be closely related to the metastasis of non-small cell lung cancer in non-small cell lung cancer patients. Later, evidences showed that MALAT1 was significantly increased in several tumors, including breast cancer [43]. Nuclear MALAT1 is involved in its proliferation, invasion, and migration of cancer cells.MALAT1 is involved in regulation of alternative splicing of mRNA precursors. The high expression of MALAT1 can promote the proliferation of tumor cells in vitro and the formation of tumors in nude mice. Knockdown of MALAT1 tumor cells greatly reduces its tumorigenicity [44].

\subsection{LSINCT5}

LSINCT5 is a long non-coding RNA with a length of $2.6 \mathrm{~kb}$ located between the IRX2 and IRX4 genes. Unlike most LncRNA, it is formed by RNA polymerase III transcription. Studies have found that the expression of 
LSINCTs is increased in some lung and breast cancer cell lines [45]. The researchers further verified the overexpression of LSINCT5 in breast and ovarian cancer cells and their tumor tissues.

LSINCT5 is over-expressed in breast cancer tissues and cell lines. Experimental research also confirmed that the expression of LSCINC5 in breast cancer cell lines and primary breast tumor tissues increased 10-fold and 7-fold compared to normal cells and breast tissues, respectively [46]. Previous research has shown that decreased expression of LSCINC5 may alter the expression levels of multiple genes, so lncRNA plays an important role in the process of cell proliferation. Besides, lncRNA NEAT1 plays a vital role in maintaining stem cell pluripotency.

\subsection{SRA}

SRA is one of the first non-coding RNAs to be discovered, and it can selectively regulate the functions of steroid receptors, such as the estrogen receptor and progesterone receptor [47]. Bioinformatics analysis shows that most of the genes affected by SRA are closely related to cell proliferation and apoptosis [48]. In addition, SRA binds to RNA helicase p68 and is involved in CTCF-mediated chromosomal recombination [49]. There are several mechanisms that modulate the function of SRA, one of which is the pseudouridine synthase family, Pus1p and Pus3p. They control the function of synergistic activation or inhibition by changing the structure of SRA [50].

SRA levels in normal breast tissue are much lower than that in tumor tissue. Some single nucleotide polymorphisms on the SRA sequence (rs10463297, rs801460) has been identified as increasing the risk of breast cancer [51]. SRA not only acts as a regulatory lncRNA, but also encodes the SRA protein (SRAP), which is highly expressed in primary breast tumors [52].

\section{Conclusion}

IncRNAs play an important role in the occurrence, metastasis, treatment and prognosis of various cancers including breast cancer. Therefore, lncRNAs have become a hot topic in cancer research. At present, several common lncRNAs, such as HOTAIR, H19, GAS5, MALAT1, LSINCT5, and SRA, have been extensively studied, and their mechanisms in breast tumors have gradually become clear as the research progresses. However, the research of lncRNAs such as LincROR, UCA1, MEG3, and NKILA is still at an early stage, and more research is needed to clarify its mechanism in breast tumorigenesis. With the study of lncRNAs, more lncRNAs will be discovered. Although our current understanding of the molecular mechanism of lncRNA in various biological processes is still limited, we hope it can be used for tumor diagnosis, treatment and prognosis for patients with breast cancer in future clinic work.

\section{References}

[1] Lei Fan, Kathrin Strasser-Weippl, Jun-Jie Li, et al. (2014). Breast cancer in China [J]. The Lancet Oncology, 15(7): e279-e289.

[2] W. Chen, R. Zheng, P. D. Baade, et al. (2016). Cancer statistics in China, 2015 [J]. CA Cancer J Clin, 66(2): 115-32.

[3] Timothy J. Key, Pia K. Verkasalo, Emily Banks. (2001). Epidemiology of breast cancer [J]. The Lancet Oncology, 2(3): 133-140.

[4] Ben Zhang, Alicia Beeghly-Fadiel, Jirong Long, et al. (2011). Genetic variants associated with breast-cancer risk: comprehensive research synopsis, meta-analysis, and epidemiological evidence [J]. The Lancet Oncology, 12(5): 477-488.

[5] Xu Yali, Sun Qiang, Shan Guangliang, et al. (2012). Risk Factors of Breast Cancer in China: A Case-Control Study [J]. Medical Journal of Peking Union Medical College Hospital, 01: 7-14.

[6] J. S. Mattick, I. V. Makunin. (2006). Non-coding RNA [J]. Hum Mol Genet, 15 Spec No 1: R17-29.

[7] A. Lujambio, S. W. Lowe. (2012). The microcosmos of cancer [J]. Nature, 482(7385): 347-55.

[8] E. Berezikov. (2011). Evolution of microRNA diversity and regulation in animals [J]. Nat Rev Genet, 12(12): 846-60.

[9] J. E. Wilusz, H. Sunwoo, D. L. Spector. (2009). Long noncoding RNAs: functional surprises from the RNA world [J]. Genes Dev, 23(13): 1494-504.

[10] R. J. Taft, M. Pheasant, J. S. Mattick. (2007). The relationship between non-protein-coding DNA and eukaryotic complexity [J]. Bioessays, 29(3): 288-99.

[11] J. Cao, C. Luo, R. Peng, et al. (2016). MiRNA-binding site functional polymorphisms in DNA repair genes RAD51, RAD52, and XRCC2 and breast cancer risk in Chinese population [J].Tumor Biol, 2016.

[12] J. Cao, C. Luo, R. Yan, et al. (2016). rs15869 at miRNA binding site in BRCA2 is associated with breast cancer susceptibility [J]. Med Oncol, 33(12): 135.

[13] J. S. Mattick. (2009). The genetic signatures of noncoding RNAs [J]. PLoS Genet, 5(4): e1000459.

[14] M. C. Lai, Z. Yang, L. Zhou, et al. (2012). Long non-coding RNA MALAT-1 overexpression predicts tumor recurrence of 
hepatocellular carcinoma after liver transplantation [J]. Med Oncol, 29(3): 1810-6.

[15] C. Braconi, N. Valeri, T. Kogure, et al. (2011). Expression and functional role of a transcribed noncoding RNA with an ultraconserved element in hepatocellular carcinoma [J]. Proc Natl Acad Sci U S A, 108(2): 786-91.

[16] H. Sun, G. Wang, Y. Peng, et al. (2015). H19 lncRNA mediates 17beta-estradiol-induced cell proliferation in MCF-7 breast cancer cells [J]. Oncol Rep, 33(6): 3045-52.

[17] R. Vikram, R. Ramachandran, K. S. Abdul. (2014). Functional significance of long non-coding RNAs in breast cancer [J]. Breast Cancer, 21(5): 515-21.

[18] Y. Li, X. Wang. (2016). Role of long noncoding RNAs in malignant disease (Review) [J]. Mol Med Rep, 13(2): $1463-9$.

[19] M. Guttman, I. Amit, M. Garber, et al. (2009). Chromatin signature reveals over a thousand highly conserved large non-coding RNAs in mammals [J]. Nature, 458(7235): 223-7.

[20] T. Derrien, R. Johnson, G. Bussotti, et al. (2012). The GENCODE v7 catalog of human long noncoding RNAs: analysis of their gene structure, evolution, and expression [J]. Genome Res, 22(9): 1775-89.

[21] S. J. Andrews, J. A. Rothnagel. (2014). Emerging evidence for functional peptides encoded by short open reading frames [J]. Nat Rev Genet, 15(3): 193-204.

[22] A. I. Nesvizhskii. (2014). Proteogenomics: concepts, applications and computational strategies [J]. Nat Methods, 11(11): 1114-25.

[23] P. J. Batista, H. Y. Chang. Long noncoding RNAs: cellular address codes in development and disease [J]. Cell, 2013, 152(6): 1298-307.

[24] Xia Tian, Xiao Bingxiu, Guo Junming. (2013). Mechanism of Long-chain Noncoding RNA and Its Research Methods [J]. Heredity, 03: 269-280.

[25] J. L. Rinn, M. Kertesz, J. K. Wang, et al. (2007). Functional demarcation of active and silent chromatin domains in human HOX loci by noncoding RNAs [J]. Cell, 129(7): 1311-23.

[26] R. A. Gupta, N. Shah, K. C. Wang, et al. (2010). Long non-coding RNA HOTAIR reprograms chromatin state to promote cancer metastasis [J]. Nature, 464(7291): 1071-6.

[27] K. M. Chisholm, Y. Wan, R. Li, et al. (2012). Detection of long non-coding RNA in archival tissue: correlation with polycomb protein expression in primary and metastatic breast carcinoma [J]. PLoS One, 7(10): e47998.

[28] M. C. Tsai, O. Manor, Y. Wan, et al. (2010). Long noncoding RNA as modular scaffold of histone modification complexes [J]. Science, 329(5992): 689-93.

[29] S. Bayram, A. T. Sumbul, C. Y. Batmaci, et al. (2015). Effect of HOTAIR rs920778 polymorphism on breast cancer susceptibility and clinicopathologic features in a Turkish population [J]. Tumour Biol, 36(5): 3863-70.

[30] R. Yan, J. Cao, C. Song, et al. (2015). Polymorphisms in lncRNA HOTAIR and susceptibility to breast cancer in a Chinese population [J]. Cancer Epidemiol, 39(6): 978-85.

[31] Y. Zhang, B. Tycko. (1992). Monoallelic expression of the human H19 gene [J]. Nat Genet, 1(1): 40-4.

[32] K. Hibi, H. Nakamura, A. Hirai, et al. (1996). Loss of H19 imprinting in esophageal cancer [J]. Cancer Res, 56(3): 480-2.

[33] Y. Fellig, I. Ariel, P. Ohana, et al. (2005). H19 expression in hepatic metastases from a range of human carcinomas [J]. J Clin Pathol, 58(10): 1064-8.

[34] C. Vennin, N. Spruyt, F. Dahmani, et al. (2015). H19 non coding RNA-derived miR-675 enhances tumorigenesis and metastasis of breast cancer cells by downregulating c-Cbl and Cbl-b [J]. Oncotarget, 6(30): 29209-23.

[35] N. Berteaux, S. Lottin, D. Monte, et al. (2005). H19 mRNA-like noncoding RNA promotes breast cancer cell proliferation through positive control by E2F1 [J]. J Biol Chem, 280(33): 29625-36.

[36] Jun Lv, Ya-Qun Yu, Shu-Qun Li, et al. (2014). Aflatoxin B1 Promotes Cell Growth and Invasion in Hepatocellular Carcinoma HepG2 Cells through H19 and E2F1 [J]. Asian Pacific Journal of Cancer Prevention, 15(6): 2565-2570.

[37] W. P. Tsang, E. K. Ng, S. S. Ng, et al. (2010). Oncofetal H19-derived miR-675 regulates tumor suppressor RB in human colorectal cancer [J]. Carcinogenesis, 31(3): 350-8.

[38] C. Ma, X. Shi, Q. Zhu, et al. (2016). The growth arrest-specific transcript 5 (GAS5): a pivotal tumor suppressor long noncoding RNA in human cancers [J]. Tumour Biol, 37(2): 1437-44.

[39] M. Mourtada-Maarabouni, M. R. Pickard, V. L. Hedge, et al. (2009). GAS5, a non-protein-coding RNA, controls apoptosis and is downregulated in breast cancer [J]. Oncogene, 28(2): 195-208.

[40] M. R. Pickard, G. T. Williams. (2014). Regulation of apoptosis by long non-coding RNA GAS5 in breast cancer cells: implications for chemotherapy [J]. Breast Cancer Res Treat, 145(2): 359-70.

[41] W. Li, L. Zhai, H. Wang, et al. (2016). Downregulation of LncRNA GAS5 causes trastuzumab resistance in breast cancer [J]. Oncotarget, 7(19): 27778-86.

[42] Z. Zhang, Z. Zhu, K. Watabe, et al. (2013). Negative regulation of lncRNA GAS5 by miR-21 [J]. Cell Death Differ, 20(11): 
1558-68.

[43] A. Guffanti, M. Iacono, P. Pelucchi, et al. (2009). A transcriptional sketch of a primary human breast cancer by 454 deep sequencing [J]. BMC Genomics, 10: 163.

[44] T. Gutschner, M. Hammerle, M. Eissmann, et al. (2013). The noncoding RNA MALAT1 is a critical regulator of the metastasis phenotype of lung cancer cells [J]. Cancer Res, 73(3): 1180-9.

[45] J. M. Silva, D. S. Perez, J. R. Pritchett, et al. (2010). Identification of long stress-induced non-coding transcripts that have altered expression in cancer [J]. Genomics, 95(6): 355-62.

[46] J. M. Silva, N. J. Boczek, M. W. Berres, et al. (2011). LSINCT5 is overexpressed in breast and ovarian cancer and affects cellular proliferation [J]. Rna Biology, 8(3): 496-505.

[47] C. M. Klinge, S. C. Jernigan, K. A. Mattingly, et al. (2004). Estrogen response element-dependent regulation of transcriptional activation of estrogen receptors alpha and beta by coactivatorsand corepressors [J]. J Mol Endocrinol, 33(2): 387-410.

[48] R. B. Lanz, S. S. Chua, N. Barron, et al. (2003). Steroid Receptor RNA Activator Stimulates Proliferation as Well as Apoptosis In Vivo [J]. Molecular and Cellular Biology, 23(20): 7163-7176.

[49] H. Yao, K. Brick, Y. Evrard, et al. (2010). Mediation of CTCF transcriptional insulation by DEAD-box RNA-binding protein p68 and steroid receptor RNA activator SRA [J]. Genes Dev, 24(22): 2543-55.

[50] Xiansi Zhao, Jeffrey R. Patton, Sajal K. Ghosh, et al. (2007). Pus3p-and pus1p-dependentpseudouridylation of steroid receptor RNA activator controls a functional switch thatregulates nuclear receptor signaling [J]. Molecular Endocrinology, 21(3): 686-699.

[51] R. Yan, K. Wang, R. Peng, et al. (2016). Genetic variants in lncRNA SRA and risk of breast cancer [J]. Oncotarget, 7: 22486-22496

[52] E. Emberley, G. J. Huang, M. K. Hamedani, et al. (2003). Identification of new human coding steroid receptor RNA activator isoforms [J]. Biochem Biophys Res Commun, 301(2): 509-15. 